\title{
Any flow is an orbit factor of any flow
}

\author{
DONALD ORNSTEIN AND BENJAMIN WEISS \\ Department of Mathematics, Stanford University, Stanford CA 94305, USA; \\ Department of Mathematics, Hebrew University, Jerusalem, Israel
}

(Received 21 July 1982)

Abstract. We prove that given any two ergodic non-singular flows $\bar{S}_{t}, S_{t}$, the first can be time changed to $\tilde{S}_{t}$ so that $S_{t}$ is a factor of $\tilde{S}_{t}$. A corresponding result for transformations is that if $\bar{T}, T$ are any two ergodic non-singular transformations then there is a tower over $\bar{T}$ that has $T$ as a factor.

\section{Introduction}

We study the question of when the flow $\left(X, S_{t}\right)$ is the orbit factor of $\left(\bar{X}, \bar{S}_{t}\right)$ in the sense that there is a (possibly many to one) $\operatorname{map} \varphi$ from $\bar{X}$ onto $X$ that maps each orbit of $\bar{S}_{t}$ diffeomorphically with positive derivative onto an orbit of $S_{t}$. If we add the requirement that the derivative of $\varphi$ be integrable then entropy theory says that there are three kinds of flows, namely those with zero, positive or infinite entropy, and the entropy cannot increase when passing to an orbit factor. The more refined equivalence theory (see [5]) gives in this case the further information that on the one hand any such orbit factor of a loosely Bernoulli (LB) flow is again LB, and on the other hand the LB flows are universal orbit factors in the sense that if $S_{t}$ is $\mathrm{LB}$ and $\bar{S}_{t}$ is arbitrary (consistent with the entropy restriction) then $S_{t}$ is an orbit factor of $\bar{S}_{t}$. (The LB flows are those that are obtained by time changing the Kronecker flow.)

The point of this paper is to show that if we drop the requirement of integrability of the derivative then any flow can map onto any other flow. Indeed the result is more general in that we do not even need to restrict to the class of finite measure preserving (f.m.p.) flows, but can encompass all ergodic non-singular flows.

Another way to describe orbit factors is as follows: $S_{t}$ is an orbit factor of $\bar{S}_{t}$ if $\bar{S}_{t}$ can be time changed to $\tilde{S}_{t}$ so that $S_{t}$ is a factor of $\tilde{S}_{t}$. Here, by a time change $\tilde{S}_{t}$, we mean that $\overline{S_{t}}$ and $\tilde{S}_{t}$ have the same orbits, and $S_{t}$ is a factor of $\tilde{S}_{t}$ means that there is a map $\psi$ from $\bar{X}$ onto $X$ so that $S_{t} \psi=\psi \tilde{S}_{t}$. Now the condition on the integrability of the derivative is equivalent to the requirement that $\tilde{S}_{t}$, the time changed $\bar{S}_{t}$, also preserves a finite measure. In this set up the above results from the equivalence theory can be formulated as follows.

1. Factors of LB flows are LB.

2. Any flow $\bar{S}_{t}$, can be time changed to a flow having LB factors.

Our main result is that we can take any flow $\bar{S}_{t}$, time change it (to a flow that, in general, will not preserve a finite measure) and get as a factor an arbitrarily pre-assigned flow $S_{t}$. Borrowing terminology from Ya. G. Sinai we get as a corollary that any two flows are weakly orbit equivalent, since each is an orbit factor of the 
other. In his basic paper [3], S. Kakutani raised the possibility that any two flows are orbit equivalent. Although this is now known not to be the case, our result shows that such speculations were not entirely vacuous. The result should also be viewed in the light of $D$. Rudolph's theorem [6] that for $\mathbb{R}^{d}$-flows, $d \geq 2$, any two ergodic f.m.p. flows are orbit equivalent. Here we have in the $\mathbb{R}^{1}$ case an inkling of the higher dimensional phenomena.

The results described above are equivalent to results concerning transformations where 'time changes' are replaced by Kakutani equivalence. Here we actually prove a stronger result - it turns out that one has only to build towers. Since we will carry out the proof in the language of transformations we formulate now our main result in that language and then indicate how to deduce the flow results.

MAIN THEOREM. If $(X, \mathscr{B}, \mu, T),(Y, \mathscr{C}, \nu, S)$ are two ergodic non-singular invertible transformations then there is a tower over $T, \hat{T}$, that has $S$ as a factor.

Given the main theorem if $\bar{S}_{t}, S_{t}$ are a pair of ergodic non-singular flows we proceed as follows: let $S$ be a cross section map for $S_{t}$, and $T$ a cross section map for $\overline{S_{t}}$ with return time bounded away from zero. By the main theorem find a tower $\hat{T}$ over $T$ that has $S$ as a factor. Realize $\hat{T}$ as a cross section map for $\bar{S}_{t}$ and then time change $\bar{S}_{t}$ to $\bar{S}_{t}^{\prime}$ so that the return time over $\hat{T}$ is constant, say equal to one. Now time change $S_{t}$ to $S_{t}^{\prime}$ so that for $S_{t}^{\prime}$ the return time over $S$ is also a constant equal to one. The fact that $\hat{T}$ has $S$ as a factor translates now to the fact that the flow $S_{t}^{\prime}$ is a factor of $\overline{S_{t}^{\prime}}$. Now change $S_{t}^{\prime}$ back to $S_{t}$, pull this change through the factor map to get a time change of $\bar{S}_{t}, \tilde{S}_{t}$, that has $S_{t}$ as a factor. We obtain as a corollary the result advertised in the title:

Corollary. Any ergodic flow $S_{t}$ is the orbit factor of any other ergodic flow.

Needless to say, to avoid trivial exceptions we are dealing only with properly ergodic flows. In our original proof of the main theorem we obtained a crucial special case by means of a new method of constructing cocycles. F. Ledrappier pointed out to us a way of avoiding the need for this construction, but because of its further developments we will nonetheless include in a final section a sketch of the original proof. We defer a more detailed description to that section. In $\S 2$ we give the proof of the main theorem for the case of f.m.p. transformations $S$. The proof for this case is elementary in that no special tools are required. In $\S 3$ we do the general case - there some familiarity with Krieger's classification of non-singular transformations appears to be necessary. The final $\S 4$ we devote to the construction of cocycles.

A final remark concerns the general phenomenon of factors. If $T$ preserves a finite measure then so does any factor, thus a factor of a type $I I_{1}$ is of type $I I_{1}$. However, already for type $I I_{\infty}$ i.e. $T$ with infinite invariant measure, a factor of $T$ may be of type III. It is of course this possibility that underlies the proof of the main theorem. H. Furstenberg pointed out to us another context where taking factors can 'complicate' matters quite a bit. The context is that of hyperfiniteness, and his example is one of a hyperfinite group action with a factor that is not hyperfinite. Let $\operatorname{SL}(3, \mathbb{Z})$ act on the flag manifold of $\operatorname{SL}(3, \mathbb{R})$. Since the stability 
group is solvable the action is amenable and hence hyperfinite, [2]. This has a factor, the same $\operatorname{SL}(3, \mathbb{Z})$ acting now on projective space. The stability group is no longer amenable, and since $\operatorname{SL}(3, \mathbb{Z})$ has finite co-volume this means that the action is no longer amenable and thus not hyperfinite.

Acknowledgements. This work was done during the ergodic theory workshop (1980), sponsored by the Institute of Advanced Studies of the Hebrew University, Jerusalem, and we benefited greatly from discussions with the participants especially J. Feldman, H. Furstenberg, Y. Katznelson and D. Rudolph. To Y. Katznelson we also owe a more specific debt for his help in what we describe in $\S 3$, and conversations with E. Lehrer led to an improvement in the exposition of $\S 4$.

\section{The invariant measure case}

We begin by recalling the notation for a tower built over a transformation. If $T: X \rightarrow X$ is a non-singular (n.s.) transformation of a probability space $(X, \mathscr{B}, \mu)$ and $h: X \rightarrow \mathbb{N}$ is a measurable mapping to the positive integers then

$$
X^{h}=\{(x, i): 1 \leq i \leq h(x)\}
$$

and $T^{h}: X^{h} \rightarrow X^{h}$ is defined by

$$
T^{h}(x, i)= \begin{cases}(T x, 1) & \text { if } i=h(x) \\ (x, i+1) & \text { if } i<h(x) .\end{cases}
$$

In a natural fashion one lifts the measurable structure from $X$ to $X^{h}$ and converts $X^{h}$ into a probability space so that $T^{h}$ is a non-singular mapping. Also, if $T$ is ergodic so is $T^{h}$ for any $h$. If $S$ is a factor of $T$, then we write $T \rightarrow S$. There are two ways in which $S$ can be recognized as a factor of $T$ :

(1) if $\mathscr{B}_{1} \subset \mathscr{B}$ is a $T$-invariant sub $\sigma$-algebra then $T_{1}$, which represents $T$ restricted to the probability space $\left(X, \mathscr{B}_{1}, \mu\right)$ is a factor of $T$;

(2) if $S$ is defined on $(Y, \mathscr{C}, \nu)$ and there is a map $\vartheta: X \rightarrow Y$ satisfying:

(a) $\vartheta^{-1}(\mathscr{C}) \subset \mathscr{B}$;

(b) $\vartheta \circ \mu$ is equivalent to $\nu$;

(c) $S \vartheta=\vartheta T$

then $S$ is a factor of $T$.

We note some elementary facts about towers and factors:

(1) If $T_{1} \rightarrow T_{2}$ and $T_{2} \rightarrow T_{3}$ then $T_{1} \rightarrow T_{3}$.

(2) $\left(T^{h_{1}}\right)^{h_{2}} \cong T^{h_{3}}$, with $h_{3}(x)=\sum_{i=1}^{h_{1}(x)} h_{2}(x, i)$.

(3) If $T \rightarrow S$, then for any tower built over $S$, say $S^{g}$, there is a tower over $T$, say $T^{\hat{\mathrm{s}}}$ so that $T^{\hat{\mathrm{g}}} \rightarrow S^{g}$.

Indeed for $\hat{g}$ one simply takes the pull-back of $g$ to the space of $T$.

If there exists some $h$ so that $T^{h} \rightarrow S$ then we will write $T \wedge S$. Our main theorem will then state that for arbitrary ergodic $T$ and $S, T \wedge S$.

LEMMA 1. If $T_{1} \wedge T_{2}$ and $T_{2} \wedge T_{3}$ then $T_{1} \wedge T_{3}$.

Proof. Let $h$ and $g$ be such that $T_{1}^{h} \rightarrow T_{2}$ and $T_{2}^{g} \rightarrow T_{3}$. By properties (1)-(3) above we see that $\left(T_{1}^{h}\right)^{\hat{s}} \rightarrow T_{2}^{g} \rightarrow T_{3}$, whence there exists some $\bar{h}$ with $T_{1}^{\bar{h}} \rightarrow T_{3}$ i.e. $T_{1} \leadsto T_{3}$. 
The next lemma is an observation of F. Ledrappier and will serve to simplify our original proof.

LEMMA 2. If $T$ is ergodic, and $r: X \rightarrow \mathbb{N}$ has the property that

$$
S x \stackrel{\operatorname{def}}{=} T^{r(x)}(x)
$$

defines an ergodic invertible transformation on $X$, then $S \Lambda_{\circ \rightarrow} T$.

Proof. One forms $X^{r}$ and builds $S^{r}$, and defines $\vartheta: X^{r} \rightarrow X$ by

$$
\vartheta(x, i)=T^{i-1} x \text {. }
$$

It is straightforward to check that $T \cdot \vartheta=\vartheta \cdot S^{r}$, whence $S^{r} \rightarrow T$ which gives the lemma.

It is a fact [1] that for any finite measure preserving transformations $S, T$ there is always some speedup of $T\left(T^{r(x)}(x)\right.$, as in the lemma) that is isomorphic to $S$. This, in conjunction with lemma 2 gives the main theorem for f.m.p. transformations. It is somewhat easier, however, to prove only a special case of this fact, namely when $S$ is the dyadic f.m.p. odometer, (see [7]) denoted henceforth by $D$, and then show separately that for any $T, T^{\wedge} \rightarrow D$. Let's begin with the second assertion and prove it first for the f.m.p. case.

LEMMA 3. If $T$ is f.m.p. then $T^{\wedge} D$ where $D$ is the f.m.p. odometer.

Proof. By Rohlin's lemma, there is a set $A_{1} \subset X$ such that $A_{1} \cap T A_{1}=\varnothing$ and $\mu(B)<0.1$ where $B_{1}=X \backslash\left(A_{1} \cup T A_{1}\right)$. To get a perfect tower of height 2 , as is required for $D$, add a single level above $B$, i.e. define $h_{1}$ to be 1 on $A_{1} \cup T A_{1}$ and 2 on $B$. Next look at $\left(T^{h_{1}}\right)^{2}$ as an ergodic mapping of $A_{1} \cup B_{1}$, and apply the Rohlin lemma, to find a set $A_{2} \subset A_{1} \cup B_{1}$ such that

$$
A_{2} \cap\left(T^{h_{1}}\right)^{2} A_{2}=\varnothing
$$

and if

$$
B_{2}=\left(A_{1} \cup B_{1}\right) \backslash\left(A_{2} \cup\left(T^{h_{1}}\right)^{2} A_{2}\right)
$$

then the relative measure of $B_{2}$ in $A_{1} \cup B_{1}$ is at most 0.01 . In order to make the tower of height 4 perfect we now add two levels over $T^{h_{1}} B_{2}$, i.e. define $h_{2}$ to be 1 on all of $X^{h_{1}}$ except for $T^{h_{1}} B_{2}$ where $h_{2}=3$. For $\left(T^{h_{1}}\right)^{h_{2}}$ we now have a set, namely $A_{2} \cup B_{2}$ that is the base of a perfect tower of height 4 . Furthermore, the new base of the perfect tower of height 2 , for $\left(T^{h_{1}}\right)^{h_{2}}$, namely

$$
\left(A_{2} \cup B_{2}\right) \cup\left(\left(T^{h_{1}}\right)^{h_{2}}\right)^{2}\left(A_{2} \cup B_{2}\right)
$$

differs by less than 0.01 from $A_{1} \cup B_{1}$. This procedure can be continued indefinitely, and the tower transformations $T^{h_{1}},\left(T^{h_{1}}\right)^{h_{2}}$ will converge to a transformation $T^{h}$ that has perfect towers of height $2^{k}$ for all $k$, and thus $T^{h}$ has $D$ as a factor. The argument shows that $\int h d \mu$ can be made to be as close to 1 as desired but we shall not need this refinement. 
Of course this lemma is a special case of the fact that $T \wedge R$ where $R$ is any loosely Bernoulli transformation of zero entropy ([5]). However since we now have to generalize the lemma to the case where $T$ is merely non-singular we have spelled out a proof.

Proposition 4. If $T$ is ergodic and non-singular then $T \leftrightarrow D$.

We would like to imitate the proof of lemma 3. However, when $T$ is merely n.s., in generàl the measures of the different levels of a Rohlin tower need not be equal, so that if we follow the proof of lemma 3 without change we will get as a factor the dyadic odometer with a measure which is far from the f.m.p. one. We need, therefore a sharpening of the usual Rohlin lemma for n.s. transformations.

LEMMA 5. If $T$ is aperiodic and non-singular, and for some $k \geqslant 1, B$ is a set in $X$ such that $B, T B, \ldots T^{k-1} B$ are disjoint and $\bigcup_{0}^{k-1} T^{j} B=X$, then for any $p \in \mathbb{N}$ and any $\varepsilon>0$ there is a set $A \subset B$ such that

(a) $A, T^{k} A, \ldots, T^{(p-1) k} A$ are disjoint;

(b) $\left|\mu\left(T^{j k+i} A\right)-\frac{1}{p} \mu\left(T^{i} B\right)\right|<\varepsilon \quad$ for $0 \leq i<k, 0 \leq j<p-1$;

(c) $\mu\left(\bigcup_{0}^{p k-1} T^{i} A\right)>1-\varepsilon$.

Proof. Consider first the case when $k=1$, and $B=X$. For a sufficiently large $N$ find a Rohlin tower $\left\{T^{j} \hat{A}\right\}_{0}^{N-1}$ of height $N$ so that the first $p$ levels and last $p$ levels have total measure $<\varepsilon / 10$, and such that the space is filled up to an extent $1-\varepsilon / 10$. Divide the base $\hat{A}$ into sets $A_{l}, 1 \leq l \leq L$, so that on each $T^{j} A_{l}, 0 \leq j<N, 1 \leq l \leq N$, the Radon Nikodym derivative of $T$ is within $\delta$ of being a constant value. If $\delta$ is sufficiently small, if each $A_{l}$ is divided into $p$ equal pieces $A_{l}^{i}, 1 \leq i \leq p$ then for all $0 \leq j<N-1$, the sets $T^{j} A_{l}^{i}, 1 \leq i \leq p$ have nearly equal measure and

$$
A=\bigcup_{l=1}^{L} \bigcup_{i=1}^{p}\left[\bigcup_{j=0}^{[N / p]-1} T^{j p+i} A_{l}^{i}\right.
$$

will satisfy (a)-(c).

For the general case we work with $T^{k}$ on $B$, with the following modifications:

(1) the $\varepsilon / 10$ is replaced by some $\varepsilon_{1}$ chosen so that if $\mu(C)<\varepsilon_{1}$ then

$$
\mu\left(T^{j} C\right)<\frac{\varepsilon}{10 k} \quad \text { for } 0 \leq j<k ;
$$

(2) the base $\hat{A}$ is divided into $A_{l}$ 's so that for all $0 \leq j<N k$ the Radon-Nikodym derivatives of $T$ on $T^{j} A_{l}$ are within $\delta$ of being constant.

Proof of proposition 4. Begin by finding some $A_{1}$ so that $A_{1}, T A_{1}$ are disjoint and

$$
\left|\mu\left(A_{1}\right)-\frac{1}{2}\right|<\frac{1}{100}, \quad\left|\mu\left(T A_{1}\right)-\frac{1}{2}\right|<\frac{1}{100}
$$

This is possible by lemma 5. As in the proof of lemma 3, define $h_{1}$ to be 1 on all of $X$ except for $X \backslash\left(A_{1} \cup T A_{1}\right)$ where $h_{1}$ is defined to be 2 . Now $T^{h_{1}}$ has a perfect tower of height 2 , with the measures of the two levels very close to 1 . It should now be clear how to complete the proof using lemma 5, and noticing that if at each 
stage the levels become more and more relatively equal, then the measure on $D$ will be equivalent to the f.m.p. one.

We turn now to the problem of showing that for any $T, D \wedge T$. According to lemma 2 what we have to do is find some $r: X \rightarrow \mathbb{N}$ so that $S x=T^{r(x)}(x)$ is isomorphic to $D$. Once again we begin with the f.m.p. case.

Proposition 6. If Tis ergodic and f.m.p. then there is some $r: X \rightarrow \mathbb{N}$ so that $T^{r(x)}(x)$ defines an invertible transformation which is isomorphic to $D$.

Proof. Recall that if $E$ and $F$ are any two sets in $X$ with the same measure there is some mapping $f: E \rightarrow \mathbb{N}$ so that if $S$ is defined on $E$ by $S x=T^{f(x)}(x)$ then $S(E)=F$. This is seen by a standard exhaustion argument (c.f. [7]). Let $\mathscr{P}_{n}$ be an increasing sequence of partitions of $X$ so that $\bigcup_{1}^{\infty} \mathscr{P}_{n}$ generates the $\sigma$-algebra $\mathscr{B}$. To start, let $A_{0}, A_{1}$ be the sets of the partition $\mathscr{P}_{1}$, and find $f_{1}$ so that $T^{f_{1}(x)}(x)=S_{1}$ maps $A_{0}$ onto $A_{1}$. Next find some partition of $A_{1}$ into $2^{k_{1}}$ sets of equal measure, $B_{i}, 1 \leq i \leq 2^{k_{1}}$, so that the $B_{i}$ 's and $S_{1}^{-1} B_{i}$ 's together approximate the sets of $\mathscr{P}_{2}$ to within $1 / 100$. Now define $\hat{S}_{2}$ on the $B_{i}$ 's for $1 \leq i \leq 2^{k_{1}}-1$ so that $\hat{S}_{2} B_{i}=S_{1}^{-1} B_{i+1}$, and on each of these $B_{i}$ 's $\hat{S}_{2}$ is of the form $T^{f_{2}(x)}(x)$. The mapping $S_{2}=S_{1} \cup \hat{S}_{2}$ is defined on all of $X$ except for $B_{2} k_{1}$, and we have now a perfect tower of height $2^{k_{1}+1}$ whose levels approximate $\mathscr{P}_{2}$ to within $1 / 100$, and a perfect tower of height 2 whose levels are $\mathscr{P}_{1}$. Now divide $B_{2} k_{1}$ into $2^{k_{2}}$ sets of equal measure, $C_{i}, 1 \leq i \leq 2^{k_{2}}$, so that the sets $S_{2}^{-j} C_{i}, 1 \leq i \leq 2^{k_{2}}, 0 \leq j<2^{k_{1}+1}$, approximate the sets of $\mathscr{P}_{3}$ to within 1/1000. Define $\hat{S}_{3}$ on the $C_{i}$ 's for $1 \leq i \leq 2^{k_{2}}-1$ so that $\hat{S}_{3} C_{i}=S_{2}^{-\left(2^{k_{1}+1}-1\right)} C_{i+1}$, and so that on each of these $C_{i}, \hat{S}_{3}$ is of the form $T^{f_{3}(x)}(x)$. Continuing this procedure we define $f=f_{1} \cup f_{2} \cup \cdots$ on almost every point of $X$, and $T^{f(x)}(x)$ is isomorphic to $D$, since the levels of the perfect towers for it approximate $\mathscr{P}_{n}$ better and better.

Combining proposition 6 with lemma 2 and proposition 4 we have proved:

Proposition 7. If $T$ is ergodic and n.s. while $S$ is ergodic and f.m.p. then $T \wedge S$.

\section{The type III case}

Since any ergodic infinite m.p. transformation $S$ can be obtained as a tower over a finite m.p. $S_{1}$ (take for $S_{1}$ the transformation induced by $S$ on a set of finite measure) we can trivially extend this last proposition to $S$ measure preserving-finite or infinite. To show that any n.s. $S$ can be allowed as the 'target' we are forced to use some results from Krieger's classification of n.s. transformations. Our strategy is to show that any n.s. transformation is actually a factor of a measure preserving one. We henceforth assume some familiarity with Krieger's theory, for which the reader may consult [7] and the references therein.

Begin by forming for any n.s. $(Y, \nu, S)$ the skew product

$$
\hat{S}(y, t)=(S y, \varphi(y) t), \quad y \in Y, t \in \mathbb{R}^{+}=(0,+\infty) .
$$

where $\varphi(y)$ is the Radon-Nikodym derivative $d \nu / d\left(S^{-1} \bullet \nu\right)$. Then $\hat{S}$ preserves the measure $\nu \times \lambda$, where $\lambda$ is Lebesgue measure on $\mathbb{R}^{+}$. The transformation $\hat{S}$ has $S$ as a factor. If $S$ is of type $I I I_{1}$ then $\hat{S}$ is ergodic, and thus we have already achieved 
our goal. If $S$ is of type $I I_{\lambda}, 0<\lambda<1$, then the ergodic components of $\hat{S}$ project onto $Y$, and we can restrict $\hat{S}$ to any such ergodic components and once again we have $S$ as a factor of an ergodic m.p. transformation. In the case where $S$ is of type $I I I_{0}$, the ergodic components of $\hat{S}$ do not project onto $Y$ and we have to make use of a further construction. We benefited greatly from conversations with $Y$. Katznelson in formulating the next few results. We first show that it suffices to work with some element orbit equivalent to $S$.

Lemma 8. If $T \rightarrow S$ and $S_{1}$ is orbit equivalent to $S$ then there is an element $T_{1}$ orbit equivalent to $T$ so that $T_{1} \rightarrow S_{1}$.

Proof. Suppose $\vartheta: X \rightarrow Y$ implements $T \rightarrow S$. If $n: Y \rightarrow \mathbb{Z}$ defines $S_{1}$, i.e. $S_{1}(y)=$ $S^{n(y)}(y)$ then $T_{1}(x)=T^{n(\vartheta x)}(x)$ is clearly in the full group of $T$, and $\vartheta \cdot T_{1}=S_{1} \cdot \vartheta$, thus $T_{1} \rightarrow S_{1}$. Furthermore since we can go back from $S_{1}$ to $S$ (recall that we assume $S_{1}$ orbit equivalent to $T_{1}$ ) we can go back in the same way from $T_{1}$ to $T$ and thus $T_{1}$ is orbit equivalent to $T$. In particular, if $T$ is ergodic so is $T_{1}$.

Our strategy is as follows: if $S_{1}$ is an arbitrary type $I I_{0}$ transformation take $S_{2}$, orbit equivalent to it, with $S_{2}$ a triadic odometer. Take for $D_{1 / 2}$ the dyadic odometer of type $I I_{1 / 2}$, given by the product measure $\Pi_{1}^{\infty}\left(\frac{1}{3}, \frac{2}{3}\right)$. Finally we shall show how to modify $S_{2}$ to an equivalent $S$ so that $S \times D_{1 / 2}$ is both ergodic and not of type $I I I_{0}$. Since obviously $S \times D_{1 / 2} \rightarrow S$ this will complete the proof of the main theorem.

To obtain $S$ from $S_{2}$ we will add measure preserving digits. Explicitly if $S_{2}$ is the odometer on $\prod_{1}^{\infty} E_{i}=Y_{2}$ with each $E_{i}=\{0,1,2\}$ we will add to $Y_{2}$ further digits as follows:

$$
Y=E_{1} \times F_{1} \times \cdots \times F_{n_{1}} \times E_{2} \times F_{n_{1}+1} \times \cdots \times F_{n_{2}} \times E_{3} \times F_{n_{2}+1} \times \cdots \times F_{n_{3}} \times E_{4} \times \cdots
$$

where $n_{1} \leq n_{2} \leq \cdots$ and if $n_{i}=n_{i+1}$ it means that no digits were added between $E_{i+1}$ and $E_{i+2}$, and each $F_{i}$ is also $\{0,1,2\}$. As far as the measure on $Y$ goes it is simply the old measure of $S_{2}$ on $Y_{2}$, with all the new digits, the $F_{i}$ 's, being independent of each other and of the old digits and identically distributed with distribution $\left(\frac{1}{3}, \frac{1}{3}, \frac{1}{3}\right)$.

No matter how the $n_{j}$ 's are chosen, $S$ so defined is orbit equivalent to $S_{2}$. This is proved in [7] for the case that $S_{2}$ is of product type. It is also true in general, and may be seen by looking at the Krieger flow associated with a type $I I_{0}$ transformation (c.f. [4]). Our claim is that if the $n_{j}$ 's grow sufficiently rapidly then $S \times D_{1 / 2}$ is ergodic and contains $\frac{1}{2}$ in its ratio set and hence is not of type $I I I_{0}$. To see this let's begin with a lemma.

LeMma 9. For any odd integer $N,\left(D_{1 / 2}\right)^{N}=T$ is ergodic and of type $I I_{1 / 2}$.

Proof. (1) Suppose that $T$ were not ergodic, and let $E \subset \prod_{1}^{\infty}\{0,1\}$ be a set with positive measure invariant under $T$. Since $E$ is measurable, it can be approximated by finite cylinder sets, and in particular, if $[w]$ for $w \in \Pi_{1}^{n}\{0,1\}$ denotes the set of points whose first $n$ coordinates are $\boldsymbol{w}$, then for $n$ sufficiently large, almost all of each such [w] are contained either in $E$ or in $E^{c}$. Furthermore, if we number these [w]'s in the natural order from $0,1, \ldots, 2^{n}-1$ then $T$ maps the $i$ 'th set onto the $i+N$ 'th set with a constant Radon-Nikodym derivative for all $i \leq 2^{n}-1-N$. Thus, with relatively few exceptions, the sets [w] can be marked as belonging to $E$ or $E^{c}$ 
and this occurs with a period of $N$. The next odometer digit divides each such [w] into two sets whose measures differ by a factor of 2 . Furthermore, considering the sets [w0] and [w1] and seeing how $T$ maps them we again get constant RadonNikodym derivatives. But since $N$ is odd the periodicity in belonging to $E$ or not belonging to $E$ will be violated as we continue past $2^{n}-1$ to $2^{n+1}-N$, unless almost everything belongs to $E$. This proves that $T$ is ergodic.

(2) To see that $\frac{1}{2}$ is in the ratio set of $T$, observe first that for any integer $N$ there is some $a \in \mathbb{Z}$ such that the number of 1 's in the binary expansion of $N+a$ is one less than the number of 1 's in the binary expansion of $a$. Indeed if $N=\sum_{i=1}^{M} 2^{k_{i}}$, with $k_{1}<k_{2}<k_{3}<\cdots<k_{M}$ then $a=\sum_{j=k_{M}}^{k_{M}+M}$ will serve since $N+a=$ $\left(\sum_{i=1}^{M-1} 2^{k_{i}}+2^{k_{M}+M+1}\right)$ has $M 1$ 's in its binary expansion while $a$ has $M+11$ 's. Now given any set $F \subset \prod_{1}^{\infty}\{0,1\}$ find some atom [w], $w \in \prod_{1}^{n}\{0,1\}$, so that the relative measure of $F$ in [w] is at least $1-\varepsilon$. Let $w^{\prime}=w 0^{k_{M}} 1^{M+1} 0$, then $T^{2^{n}}\left[\mathrm{w}^{\prime}\right]=\left[\mathrm{w}^{\prime \prime}\right]$, where $w^{\prime \prime}=w v$ and $v$ is the binary representation of $N+a$. It follows that $T^{2^{n}}$ maps [ $\left.\mathrm{w}^{\prime}\right]$ onto $\left[w^{\prime \prime}\right]$ with Radon-Nikodym derivative $\frac{1}{2}$. If $\varepsilon$ is small enough, the relative

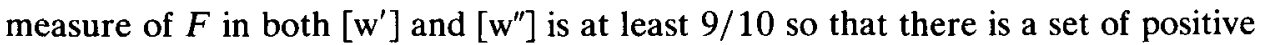
measure in $F$ that returns to $F$ under $T^{2^{n}}$ with Radon-Nikodym derivative $\frac{1}{2}$, hence $\frac{1}{2}$ is in the ratio set of $T$. Since clearly all Radon-Nikodym derivatives of $T$ are powers of 2 this shows that $T$ is of type $I I_{1 / 2}$.

The point of adding the measure preserving digits to form $Y$ is to give $S$ the property that $S^{j}$ acts as a measure preserving transformation on a large part of the space for $j$ equal to many multiples of $3^{k+n_{k}}$. We formalize the property as an easy lemma whose proof is left to the reader.

LEMMA 10. If $S$ is the odometer transformation on $\left(\prod_{1}^{\infty} G_{i}, \lambda\right)$ where each $G_{i}=$ $\{0,1,2\}$, and the measure $\lambda$ has the property that the coordinates $G_{n+1}, G_{n+2}, \ldots, G_{n+k}$ are independent of all the remaining coordinates, and of each other, and on each $G_{j}$, $n<j \leq n+k, \lambda$ is the measure $\left\{\frac{1}{3}, \frac{1}{3}, \frac{1}{3}\right\}$, then on each atom of $\prod_{1}^{n} G_{i}, S^{j 3^{n}}$, which fixes this atom, is measure preserving in the complement of a set whose relative measure is at most $|j| 3^{-k}$.

Now we can explain how to choose $n_{k+1}$, given $n_{1}, \ldots, n_{k}$ so that our $S$ will have the desired properties. One looks at $\left(D_{1 / 2}\right)^{N}=T$ with $N=3^{n_{k}+k+1}$ (corresponding to $\left.E_{1} \times F_{1} \times \cdots \times F_{n_{k}} \times E^{k+1}\right)$ and at the partition $\left\{A_{1}, \ldots, A^{2^{k+1}}\right\}$ corresponding to the first $k+1$ digits of the $D_{1 / 2}$ odometer. By lemma 9 , and the fact that if $T$ is an ergodic transformation of type $I I_{1 / 2}$ all of whose $\mathrm{R}-\mathrm{N}$ derivatives are powers of 2 , and $A$ and $B$ are any two sets the ratio of whose measures is a power of 2 , then there is a mapping in the groupoid of $T$ sending $A$ to $B$ with constant $\mathrm{R}-\mathrm{N}$ derivatives, we can find elements $T_{i j}$ in the groupoid of $T$ so that $T_{i j}\left(A_{i}\right)=A_{j}$, $1 \leq i<j<2^{k+1}$ where the $T_{i j}$ 's have constant $\mathrm{R}-\mathrm{N}$ derivatives. Furthermore, we can divide each $A_{i}$ into two sets $A_{i}^{\prime}$ and $A_{i}^{\prime \prime}$ with

$$
\mu\left(A_{i}^{\prime} \mid A_{i}\right)=\frac{1}{3}, \quad \mu\left(A_{i}^{\prime \prime} \mid A_{i}\right)=\frac{2}{3}
$$

and find elements in the groupoid of $T, T_{i}$, so that $T_{i}\left(A_{i}^{\prime}\right)=A_{i}^{\prime \prime}$ with $T_{i}$ having a constant $\mathrm{R}-\mathrm{N}$ derivative. 
Having found the $T_{i j}$ 's, and the $T_{i}$ 's one finds an $M$ so that these mappings can be defined using powers of $T$, between $-M$ and $M$ on all but sets with relative measure at most $10^{-k}$. This is possible simply because each $T_{i j}$ and $T_{i}$ is defined by some measurable map $u_{i j}, u_{i}$ as $T^{u_{i j}}, T^{u_{i}}$. Now, having found $M$, one chooses $n_{k+1}$ so that $M \cdot 3^{-\left(n_{k+1}-n_{k}\right)}<10^{-k}$. This completes the definition of $S$, and all that remains is to check that $S \times D_{1 / 2}$ is ergodic and contains $\frac{1}{2}$ in its ratio set.

We begin with the ergodicity. Suppose $B$ is an invariant set for $S \times D_{1 / 2}$ of positive measure. If $B_{y}$ is the section of $B$ over $y \in Y$, then since $S$ is ergodic, the measure of $B_{y}$ is constant. Since $B$ is measurable if $\varepsilon>0$, for some large enough $k$, we have some atom $C$ corresponding to $E_{1} \times F_{1} \times \cdots \times E_{k+1}$ and some $A$ corresponding to the first $k$ digits of $D_{1 / 2}$ so that $B \cap C \times A$ has relative measure at least $1-\varepsilon$. For any other $\bar{A}$, using the appropriate $T_{i j}$ 's, and lemma 10 one sees that $B \cap C \times \bar{A}$ also has relative measure at least $1-2 \varepsilon$ (if $10^{-k}<\frac{1}{10} \varepsilon$ ), and thus the sections of $B$ over most $y$ 's in $C$ is at least $1-\sqrt{2 \varepsilon}$. Since $\varepsilon$ is arbitrary we conclude that $B$ has measure 1 .

To see that $\frac{1}{2}$ is in the ratio set, let $B$ be any subset for $S \times D_{1 / 2}$ with positive measure. As before find some $A \times C$ which is almost filled up by $B$, and then use the appropriate $T_{i}$ observing that if $B$ almost fills up $A \times C$ it does the same for $A^{\prime} \times C$ and $A^{\prime \prime} \times C$. Once again lemma 10 and the choice of the $n_{k}$ 's complete the proof.

\section{Cocycles}

Our original proof of the main theorem was based on a result that is interesting in its own right which we proceed to describe. If $\alpha: X \rightarrow \mathbb{Z}$ is a measurable function then the random walk, or the cocycle, defined by $\alpha$ is:

$$
\begin{aligned}
\alpha(x, k) & =\sum_{0}^{k-1} \alpha\left(T^{j} x\right), \quad k \leq 1 \\
\alpha(x,-k) & =\sum_{1}^{k} \alpha\left(T^{-j} x\right), \quad k \geq 1 \\
\alpha(x, 0) & =0 .
\end{aligned}
$$

The skew product $T_{\alpha}$ is defined by

$$
T_{\alpha}(x, n)=(T x, n+\alpha(x))
$$

and thus is a mapping of $X \times \mathbb{Z}$ onto $X \times \mathbb{Z}$. The cocycle $\alpha(x, k)$ enters by the formula

$$
\left(T_{\alpha}\right)^{k}(x, n)=\left(T^{k} x, n+\alpha(x, k)\right),
$$

and thus the properties of the iterates of $T_{\alpha}$ depend on the properties of the cocycle. If $T_{\alpha}$ is conservative then $T_{\alpha}$ induces a transformation on $X \times\{0\}$, explicitly:

$$
\begin{gathered}
r(x)=\min \{k \geq 1: \alpha(x, k)=0\} \\
T_{\alpha}^{*}=T^{r(x)}(x) .
\end{gathered}
$$

If in addition, $T_{\alpha}$ is ergodic then $T_{\alpha}$ can be viewed as a tower built over $T_{\alpha}^{*}$. Since $T$ is manifestly a factor of $T_{\alpha}$ we have that for all $\alpha$ such that $T_{\alpha}$ is ergodic: $T_{\alpha}^{*} \wedge T$. 
It is natural then to ask what kind of transformation can $T_{\alpha}^{*}$ be for an ergodic $T_{\alpha}$. The following theorem holds:

THEOREM. If $T$ is ergodic and f.m.p., there is an $\alpha$ so that $T_{\alpha}$ is ergodic and $T_{\alpha}^{*}$ is an LB transformation of zero entropy and even of rank 1.

(For definition and properties of rank 1 see [5].)

Since any zero entropy LB is a tower over the dyadic odometer (see [5]) this theorem can replace lemma 2 and proposition 6 , and that is how the original proof went. It seems plausible that the theorem can be sharpened to the assertion that $T_{\alpha}^{*}$ can be already the dyadic odometer, or indeed any pre-assigned transformation, but we leave these questions for future investigations. The method that we developed for proving this theorem proved to be useful in answering other kinds of questions about cocycles. Recall that $a$ is a recurrent value for the cocycle $\alpha(x, k)$ if for a.e. $x$ and all $\varepsilon>0,|\alpha(x, k)-k a|<\varepsilon$ for infinitely many values of $k$. In particular, we could construct a cocyle such that 0 is the unique recurrent value but nonetheless along most $n$ 's, $(1 / n) \alpha(x, n)$ tends to $\infty$ in probability. On the other hand we could also construct examples where $(1 / n) \alpha(x, n) \rightarrow 0$ in probability, but nonetheless all values are recurrent.

Now for a sketch of the proof of the theorem. Fix first some sequence of finite partitions $\mathscr{P}_{1} \subset \mathscr{P}_{2} \subset \cdots$ so that $\bigvee_{1}^{\infty} \mathscr{P}_{j}=\mathscr{B}$, the full $\sigma$-algebra of $(X, T)$. In order to see that $T_{\alpha}^{*}$ is LB we shall construct $\alpha$ so that for all $j,\left(T_{\alpha}^{*}, \mathscr{P}_{j}\right)$ is a zero entropy LB process, and this suffices - since the inverse limit of LB processes is LB. In order to simplify the description we shall restrict attention to a single $\mathscr{P}$. The definition of $\alpha$ will be carried out in a series of steps using a nested sequence of Rohlin towers $\left\{A_{n}, h_{n}\right\}$, where

$$
T^{j} A_{n} \cap A_{n}=\varnothing \quad \text { for } 1 \leq j<h_{n}
$$

and

$$
\cdots \subset \bigcup_{k=0}^{h_{n}-1} T^{k} A_{n} \subset \bigcup_{k=0}^{h_{n+1}-1} T^{k} A_{n+1} \subset \cdots
$$

with

$$
\lim _{n \rightarrow \infty} \mu\left(\bigcup_{0}^{h_{n}-1} T_{k} A_{n}\right)=1
$$

At the end of the $n$ 'th stage, $\alpha$ will have been defined on all levels of the $n$ 'th tower - with the exception of the roof $-T^{h_{n}-1} A_{n}$. With this in mind we'll now describe the first step. Using the ergodic theorem and the strong Rohlin lemma, we can find $A_{1}$ and $h_{1}$ so that when $\left(A_{1}, h_{1}\right)$ is partitioned into pure columns with respect to $\mathscr{P}$, most of the columns have a very regular distribution of the atoms of $\mathscr{P}$. For simplicity suppose that $\mathscr{P}$ has two atoms $P_{0}, P_{1}$ of equal measure. Then we can guarantee that $h_{1}$ is of the form $k_{1} M_{1}$, and that for most of the pure columns, each block from $j k_{1}$ to $j+k_{1}-1$, for $0 \leq j<M_{1}$, has about half zeros and about half ones. If we denote a typical such name by $a_{1} a_{2} \cdots a_{k_{1} M_{1}}, a_{i} \in\{0,1\}$, then on that 
pure column $\alpha$ will be defined in such a way, that for the most part, $T_{\alpha}^{*}$ will encounter the name $010101 \cdots$ or $101010 \cdots$. We illustrate how this is done with a simple example: $k_{1}=8, M_{1}=5$, and consider the diagram:

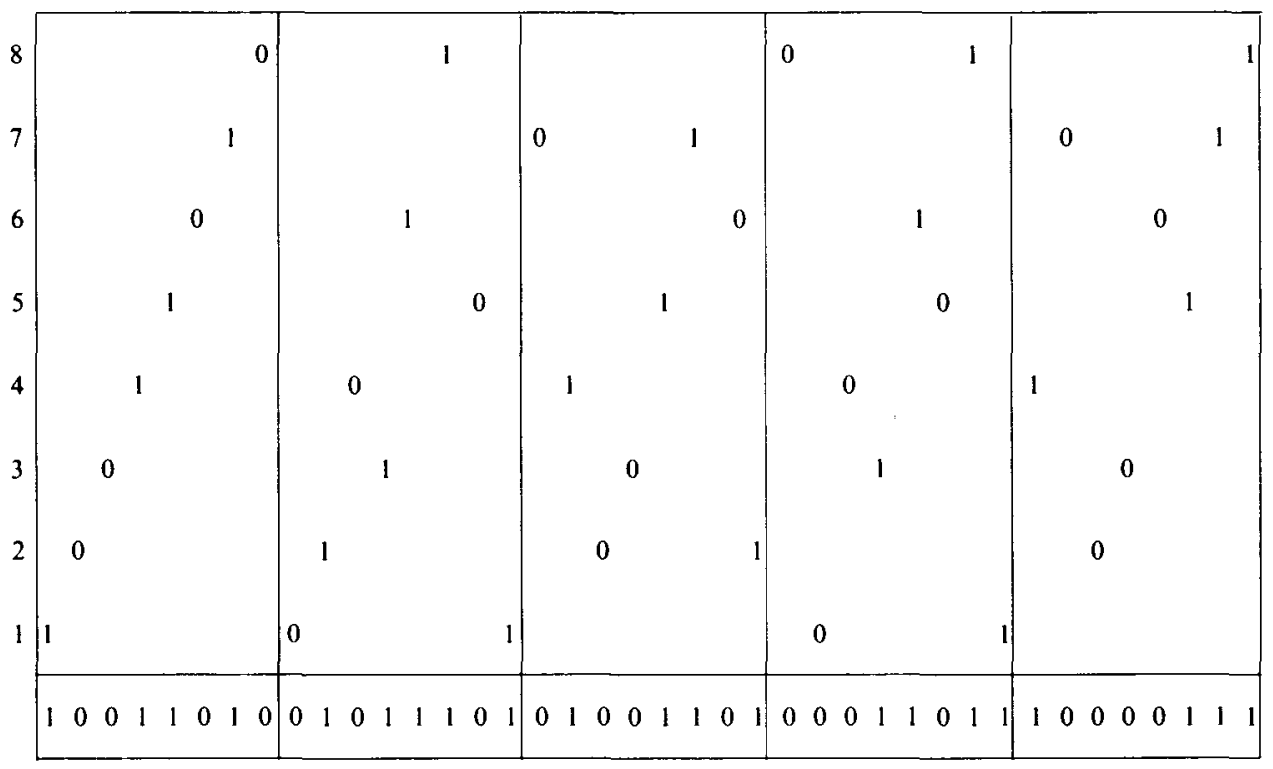

FIGURE 1

The diagram is meant to give the graph of $\alpha(x, j)$ for a point $x$ in the base of the tower. As one can see, the $T_{\alpha}^{*}$ name, which is what one reads when looking across horizontal rows is for most of the rows the periodic sequence $0101 \cdots$ or $1010 \cdots$.

On those pure columns where the distribution is not right $\alpha$ is defined in an arbitrary fashion. At the second stage, we operate in the same way, but the individual symbols are now replaced by whole blocks of length $k_{1} M_{1}$. The ergodic theorem is now applied so that for a large enough $h_{2}$ of the form $k_{2} M_{2}$, each of the $M_{2}$ blocks of length $k_{2}$ has the distribution of $h_{1}$-names being very close to their true distribution. Then once again a diagram similar to figure 1 shows how to cycle through in a periodic fashion so that horizontal lines look pretty much the same. The only slightly subtle point is the following: since we don't wish to modify the definition of $\alpha$ on the part of the space where it was defined in stage 1, we move whole $h_{1}$-blocks up and down the diagram. However, a single such block has different distribution across each of its $k_{1}$ lines. To smooth out, blocks are, repeated according to the following pattern:

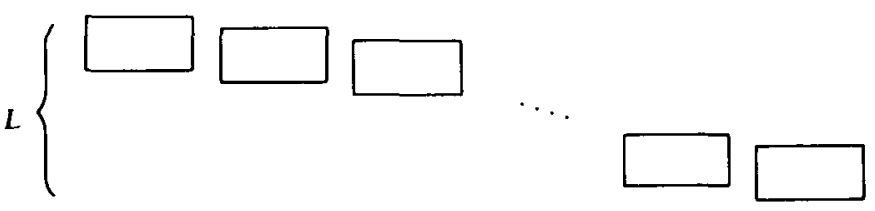

Figure 2 
where $L$ is large compared to $k_{1}$, so that on most of the $L$ lines, when the block is encountered by $T_{\alpha}^{*}$, it sees in succession the first, second, $\cdots k_{1}$ level of the same block. This simply means that in addition to other choices, $M_{2}$ must be large compared to $L$.

It should be clear by now how to force greater and greater periodicity in the names that $T_{\alpha}^{*}$ will encounter, and thus $T_{\alpha}^{*}$ will be of rank 1 . It will thus automatically be ergodic, and then it is not hard to check that $T_{\alpha}$ will also be ergodic (naturally we must be careful not to choose all values of $\alpha$ divisible by some fixed integer).

\section{REFERENCES}

[1] P. Arnoux, D. S. Ornstein \& B. Weiss. Cutting and stacking, interval exchanges and geometric models. Israel $J$. Math. To appear.

[2] A. Connes, J. Feldman \& B. Weiss. Amenable equivalent relations are generated by a single transformation. Ergod. Th. \& Dynam. Sys. 1 (1981), 431-450.

[3] S. Kakutani. Induced measure preserving transformation. Proc. Japan Acad. 19 (1943), 635-641.

[4] W. Krieger. On ergodic flows and isomorphism of factors. Math Ann. 223 (1976), 19-70.

[5] D. Ornstein, D. Rudolph \& B. Weiss. Equivalence and non equivalence theory. Memoirs Amer. Math. Soc. 262 (1982).

[6] D. Rudolph. Smooth orbit equivalence of ergodic $R^{d}$-actions, $d \geq 2$. Trans. Amer. Math. Soc. 253 (1979), 291-302.

[7] B. Weiss. Orbit equivalence of non-singular actions. In Theorie Ergodique, Monog. 29, Enseign. Math. 1981, pp. 77-107. 